\title{
Equation for the Microwave Backscatter Cross Section of Aggregate Snowflakes Using the Self-Similar Rayleigh-Gans Approximation
}

\author{
Robin J. HogAn AND CHRISTOPHER D. WeSTBROOK \\ Department of Meteorology, University of Reading, Reading, United Kingdom
}

(Manuscript received 31 October 2013, in final form 27 April 2014)

\begin{abstract}
In this paper an equation is derived for the mean backscatter cross section of an ensemble of snowflakes at centimeter and millimeter wavelengths. It uses the Rayleigh-Gans approximation, which has previously been found to be applicable at these wavelengths due to the low density of snow aggregates. Although the internal structure of an individual snowflake is random and unpredictable, the authors find from simulations of the aggregation process that their structure is "self-similar" and can be described by a power law. This enables an analytic expression to be derived for the backscatter cross section of an ensemble of particles as a function of their maximum dimension in the direction of propagation of the radiation, the volume of ice they contain, a variable describing their mean shape, and two variables describing the shape of the power spectrum. The exponent of the power law is found to be $-5 / 3$. In the case of $1-\mathrm{cm}$ snowflakes observed by a $3.2-\mathrm{mm}$ wavelength radar, the backscatter is $40-100$ times larger than that of a homogeneous ice-air spheroid with the same mass, size, and aspect ratio.
\end{abstract}

\section{Introduction}

Most surface precipitation poleward of $60^{\circ}-70^{\circ}$ latitude is in the ice phase, but devising reliable methods to estimate snowfall rate from space has proved very challenging (Levizzani et al. 2011). Recently, the 94-GHz (3.2-mm wavelength) CloudSat radar has been used to make estimates of snowfall from space (Liu 2008); this frequency has the advantage of a very high sensitivity to low snowfall rates, but these retrievals have significant uncertainties due to the uncertain backscatter cross section of snowflakes at wavelengths shorter than the size of the particle.

The state of the art in computing the backscatter cross section of snowflakes is to use the discrete dipole approximation (DDA; Draine and Flatau 1994), but this is very computationally expensive and requires a large number of realistic 3D snowflake shapes to be generated. Since aggregate snowflakes tend to have a low density at the millimeter scale, the Rayleigh-Gans approximation (RGA; van de Hulst 1957; Matrosov 1992; Westbrook et al. 2006) may be used. It is also applicable

Corresponding author address: Robin J. Hogan, Dept. of Meteorology, Earley Gate, P.O. Box 243, Reading RG6 6BB, United Kingdom.

E-mail: r.j.hogan@reading.ac.uk for light scattering by some aerosol aggregates (Sorensen 2001). The RGA approximates the electric field experienced by a dipole within the particle as the incident electric field, thereby neglecting any interaction between dipoles. This leads to a simple equation for the backscatter that requires only a $1 \mathrm{D}$ description of the structure of the particle in the direction of propagation of the incident wave. Comparisons between DDA and RGA by Tyynelä et al. (2013) and Leinonen et al. (2013) found RGA to be accurate typically to around $2 \mathrm{~dB}$. While this difference is not negligible, Leinonen et al. (2013) argued that RGA is useful as it captures the firstorder physics of the problem, providing a good estimate of backscatter cross section with the interactions between dipoles being only a relatively minor correction. Moreover, as will be shown, the differences due to different assumptions on the nature the internal structure can be much larger than $2 \mathrm{~dB}$.

In the framework of RGA, if the 1D function describing particle structure could be parameterized in some way then it would avoid the need to generate a large ensemble of 3D snowflakes. A common and simple approach to treating this function is to approximate snowflakes as "soft spheroids," that is, oblate horizontally oriented spheroids with an aspect ratio of around 0.6 comprising a homogeneous mixture of ice and air (e.g., Matrosov et al. 2005a). While this has been 
found to perform very well for aggregates in ice clouds for particle sizes no larger than the wavelength (Hogan et al. 2012), it has been found to systematically underestimate the backscatter for larger snowflakes (e.g., Petty and Huang 2010; Tyynelä et al. 2011).

In this paper, we first use numerically simulated aggregates to parameterize the mean shape of this $1 \mathrm{D}$ function describing particle structure and show that deviations from the mean have a power spectrum that follows a power law. This enables the function to be parameterized by three coefficients: one for the mean shape and two for the power law (section 2). These are then used to derive an equation for the mean backscatter cross section of an ensemble of aggregate snowflakes and validate it against the mean backscatter of the simulated aggregates (section 3). Finally, we demonstrate the impact of the new model on calculations of $94-\mathrm{GHz}$ radar reflectivity factor for realistic snow distributions (section 4). This work naturally explains why the softspheroid approach systematically underestimates the backscatter of large snowflakes. Note that comparisons of DDA against RGA are not performed, since they have been carried out in previous papers (e.g., Tyynelä et al. 2013; Leinonen et al. 2013).

\section{Statistical description of particle shape}

\section{a. The Rayleigh-Gans approximation}

In Rayleigh-Gans theory (also known as the Born approximation), the backscatter cross section (or radar cross section) of an arbitrarily shaped particle illuminated by a plane wave propagating in the direction $s$ is given by (van de Hulst 1957; Westbrook et al. 2006; Leinonen et al. 2013)

$$
\sigma_{b}=\frac{9 k^{4}|K|^{2}}{4 \pi}\left|\int_{-D / 2}^{D / 2} A(s) \exp (i 2 k s) d s\right|^{2}
$$

where $k$ is the wavenumber of the radiation and $K=$ $(\varepsilon-1) /(\varepsilon+2)$ is the Clausius-Mossotti factor with $\varepsilon$ the dielectric constant of solid ice. The maximum extent of the particle in the $s$ direction is $D$ and the $s$ coordinate is centered on the particle such that the particle lies completely within the range $-D / 2 \leq s \leq D / 2$. Variable $A(s)$ is the area of particle material intersected by a plane at range $s$. In the case of ice aggregates, $A(s)$ is the area of solid ice intersected by the plane. Naturally, $\exp (i 2 k s)$ may be replaced by $\cos (2 k s)+i \sin (2 k s)$, and in the case of particles that are symmetric about $s=0$, such as spheres and spheroids, the integral of $A(s) i \sin (2 k s)$ is zero so the complex exponential may be replaced by $\cos (2 k s)$. But in the general case it is necessary to retain the imaginary term. Note that Eq. (1) gives backscatter cross section $\left(\mathrm{m}^{2}\right)$, but in some conventions there is an extra factor of $4 \pi$ on the denominator to yield cross section per unit solid angle $\left(\mathrm{m}^{2} \mathrm{sr}^{-1}\right)$; see Bohren and Huffman (1998) for a discussion.

For soft spheres and horizontally aligned soft oblate spheroids observed by a vertical- or nadir-pointing radar, we may write $A(s)=\pi f\left(D_{\max }^{2} / 4-s^{2} / \alpha^{2}\right)$, where $f$ is the fraction of the volume of the ice-air mixture that is ice, $D_{\max }$ is the maximum extent of the particle in the horizontal dimension, and $\alpha$ is the aspect ratio such that the maximum extent of the particle in the vertical dimension is $D=\alpha D_{\text {max }}$. Substitution into Eq. (1) yields

$$
\sigma_{b}=\frac{9 \pi|K|^{2} f^{2}}{16 k^{2} \alpha^{4}}[\sin (k D)-k D \cos (k D)]^{2}
$$

In section 4, this expression for soft spheroids will be compared to the new approximation developed in this paper.

\section{b. Ensemble of simulated aggregates}

Equation (1) is easy to use for individual arbitrarily shaped particles for which $A(s)$ is known. For an ensemble of particles of the same size but with different internal structures, it is possible to laboriously compute the mean backscatter cross section by applying Eq. (1) to the $A(s)$ function of each particle separately, and averaging. A more elegant and convenient way is to describe the characteristics of $A(s)$ statistically, such that a formula can be derived for the mean backscatter cross section of an ensemble of particles.

To demonstrate how the structure of snowflakes may be characterized, we make use of three databases each of around 50 simulated aggregate snowflakes generated using the aggregation model of Westbrook et al. (2004). The aggregates in the first database comprise around 100 bullet rosettes of different sizes, where the aspect ratio of the columnar branches of the bullet rosettes is 3.5 . The second database comprises aggregates of hexagonal columns with an aspect ratio of 3.5 and the third comprises aggregates of plates with an aspect ratio of 0.2. Since ice particles fall with their maximum extent oriented horizontally (e.g., Sassen 1977; Pruppacher and Klett 1997; Hogan et al. 2002), each aggregate has been oriented such that its maximum extent lies in the $x$ direction and its minimum extent is in the $z$ direction. Matrosov et al. (2005b) reported measurements of horizontally aligned particles wobbling with a standard deviation of $9^{\circ}$. For a representative aspect ratio for aggregates of 0.6 (e.g., Matrosov et al. 2005a; Hogan et al. 2012), such wobbling only increases the extent of the particle in the vertical direction by $2 \%$, which is 


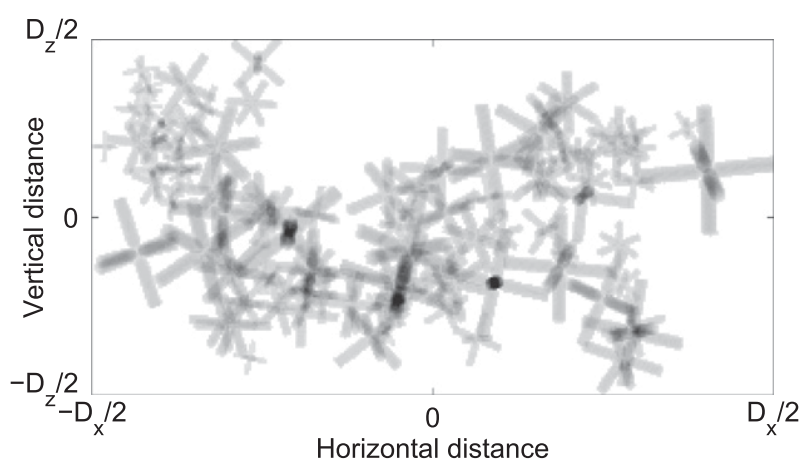

FIG. 1. Example of a simulated aggregate of bullet rosettes, shown in the plane of its longest $(x)$ dimension and shortest $(z)$ dimension, with the intensity of the shading proportional to the amount of ice in the third $(y)$ dimension. See the dotted lines in Figs. $2 \mathrm{a}$ and $2 \mathrm{~b}$ for the $A(x)$ and $A(z)$ functions for this particle.

much less than the uncertainty in the vertical dimension of the particle, so may be safely neglected.

An example of an aggregate of bullet rosettes is depicted in Fig. 1. We stress that the three databases are unlikely to capture the full diversity of real snow aggregates, but rather provide plausible models of three common types of aggregates. If the parameters characterizing aggregate structure do not vary very much between databases, then this provides confidence that the scattering properties of other types of ice aggregate in the atmosphere are likely to be similar to those reported here. Nonetheless, there would be value in applying the analysis method described in this paper to other sources of three-dimensional aggregate structure information. A further point to note is that the analysis here is independent of the absolute size of the aggregates or their mass-size relationship.

We next consider the $A(s)$ functions for the database of simulated aggregates of bullet rosettes. The dotted lines in Figs. 2a and 2b depict $A(x)$ and $A(z)$, respectively, for the aggregate shown in Fig. 1. These are the functions that would be used in Eq. (1) to compute the backscatter cross section for a radar observing the particle horizontally (parallel to the $x$ axis) and vertically. The solid black line in these figures shows these functions averaged over all 50 aggregates. In fact, the line in Fig. 2a is the average over the two horizontal dimensions [i.e. the average of $A(x)$ and $A(y)$ ], since it can be assumed that the maximum dimension of the particle, while in the horizontal plane, would have a random azimuth angle. The solid black line in Fig. 2c shows the average of $A(x), A(y)$, and $A(z)$, intended to approximately represent the shape that is relevant for random particle orientation. In each panel of Fig. 2, the gray region indicates one standard deviation above and below the mean.

An interesting aspect of the mean structure is the difference in kurtosis between Figs. 2a and 2b, with the mean structure in the vertical direction tending to be peaked strongly in the middle (positive kurtosis), while the mean structure in the horizontal direction has a much flatter distribution (negative kurtosis). This difference can be explained by considering two quasispherical aggregates (i.e., with similar $A$ functions in the vertical and horizontal) of similar size that collide to form a larger aggregate. The join point will likely be near the edge of both. The new particle will then align itself horizontally such that the centers of the original aggregates are at around the same height. The horizontal function $A(x)$ aligned along the new particle's major axis will then be very different from $A(z): A(x)$ will be broader as a result of the presence of the two original aggregates and will have a minimum somewhere close to the middle corresponding to the join point; by contrast, $A(z)$ will be an average of the structures of the two aggregates and this average will tend to smooth out fluctuations from the mean behavior.
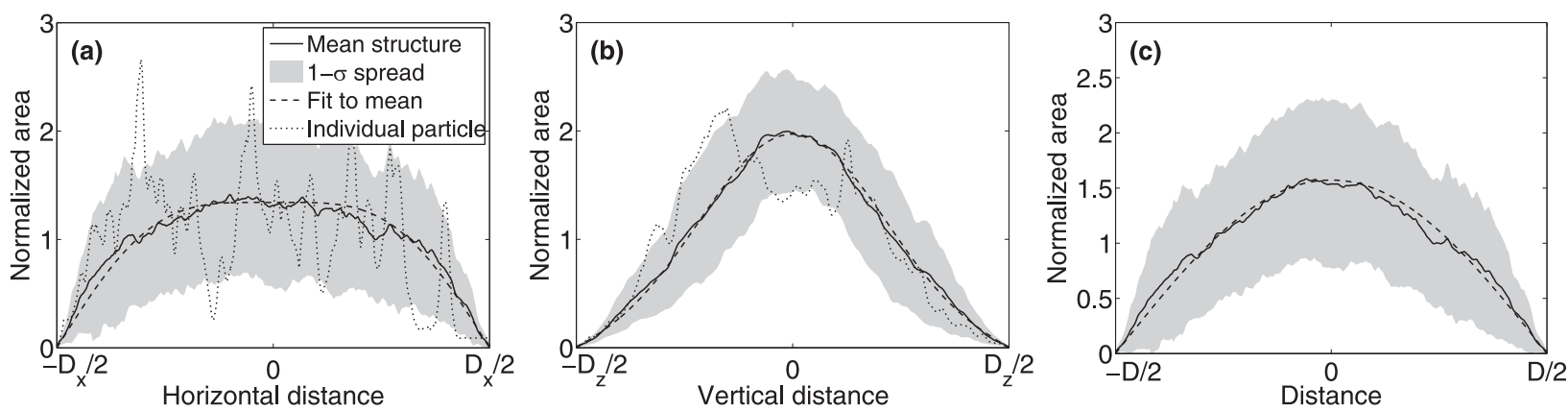

FIG. 2. The normalized area of ice, $A(s) / \overline{A(s)}$, of the ensemble of horizontally aligned aggregates of bullet rosettes discussed in section $2 \mathrm{~b}$ as a function of distance through the aggregates: (a) horizontally, (b) vertically, and (c) in a random direction. The black solid line indicates the mean of the ensemble while the gray shaded region encompasses plus or minus one standard deviation. The dashed lines show the fit to the mean represented by the first term on the right-hand side of Eq. (3) with the $\kappa$ coefficients in Table 1 . The dotted lines in (a) and (b) indicate $A(x)$ and $A(z)$, respectively, for the specific aggregate shown in Fig. 1. 


\section{c. Fourier-like decomposition of particle shape}

The next step is to parameterize both this mean shape and the fluctuations from the mean exhibited by individual particles. Most previous statistical descriptions of aggregates have been in terms of the density autocorrelation function, assuming that their internal structure is fractal and, therefore, that the autocorrelation follows a power law [see the review by Sorensen (2001)]. However, to represent the finite size of the aggregate requires the power law to be multiplied by a semiarbitrary cutoff function, and the resulting backscatter can be quite sensitive to the functional form that is chosen. Leinonen et al. (2013) described the autocorrelation function instead in terms of the sum of two Gaussians: one representing the overall aggregate and the other the individual monomers. The sharpness of the edges of a Gaussian meant that no cutoff function was required, but the two-Gaussian model does implicitly assume the monomers to be randomly distributed within the aggregate, neglecting the possibility of internal clumping at other scales, which is expected from the fractal paradigm.

Our approach is different: instead of considering the autocorrelation function, the statistical description of the ensemble of particles is obtained by performing a decomposition of $A(s)$ as follows:

$$
\begin{aligned}
A(s)= & a_{0}\left[\left(1+\frac{\kappa}{3}\right) \cos \left(\frac{\pi s}{D}\right)+\kappa \cos \left(\frac{3 \pi s}{D}\right)\right] \\
& +\sum_{j=1}^{n} a_{j}^{\prime} \cos \left(\frac{2 \pi j s}{D}\right)+a_{j}^{\prime \prime} \sin \left(\frac{2 \pi j s}{D}\right),
\end{aligned}
$$

where $A(s)$ is nonzero only in the range $\pm D / 2$. The first term on the right-hand side captures the gross structure averaged across the ensemble, while the second term represents fluctuations of an individual particle from the ensemble average in terms of a summation capturing the smaller-scale and smaller-amplitude variations. This is similar to a Fourier decomposition of a real function, except that rather than the first term on the right-hand side being constant with $s$, it has a prescribed shape expressed in terms of two cosines. The $\kappa$ coefficient controls the kurtosis of this shape while conserving total volume. The case of $\kappa=0$ leads to just a single cosine, and it can be seen from the dashed line in Fig. $2 \mathrm{c}$ that this is a very good fit to the ensemble-mean behavior for random orientation. The dashed lines in Figs. $2 \mathrm{a}$ and $2 \mathrm{~b}$ show that an equally good fit can be obtained for the ensemble-mean behavior for horizontal and vertical orientation by manipulating $\kappa$; the actual values used are shown in Table 1. This justifies the functional form within the square brackets in Eq. (3). Note that Leinonen et al.
TABLE 1. Parameters describing the structure of horizontally oriented aggregates in the horizontal dimension (relevant for horizontally pointing radars), the vertical dimension (relevant for vertically pointing radars), and without consideration of orientation or direction of incidence (relevant for randomly oriented aggregates). The kurtosis parameter describes the mean structure in Eq. (3) while the power-law prefactor describes the amplitude of the fluctuations about the mean structure in Eq. (5). Note that the parameter defining the slope of the power law in Eq. (5) has been found to have a value of $\gamma=5 / 3$ for all cases considered.

\begin{tabular}{lcc}
\hline \hline Incidence & Kurtosis parameter $\kappa$ & Power-law prefactor $\beta$ \\
\hline Aggregates of bullet rosettes or columns & \\
Horizontal & -0.11 & 0.56 \\
Vertical & 0.19 & 0.23 \\
Random & 0.00 & 0.45 \\
Aggregates of plates & & \\
Horizontal & -0.12 & 0.61 \\
Vertical & 0.18 & 0.21 \\
Random & -0.05 & 0.51 \\
\hline
\end{tabular}

(2013) instead described $A(s)$ by a Gaussian. While the Gaussian is convenient mathematically, we argue that it is less appropriate than the form given by Eq. (3) because it is nonzero for all $s$ and is a plausible fit only for the mean shape of $A(z)$ (Fig. 2b), but not the other two cases (Figs. 2a and 2c).

Table 1 also shows $\kappa$ for the other two aggregate databases. In the case of the aggregates of plates the differences are fairly small, and for aggregates of columns, $\kappa$ is indistinguishable from the value for aggregates of bullet rosettes. This is unsurprising since the individual branches of the simulated bullet rosettes had the same aspect ratio as the columns.

Once $\kappa$ is estimated from the ensemble of particles, we may perform the decomposition represented by Eq. (3) on a real particle with known $A(s)$. The coefficient of the first term is set to

$$
a_{0}=\frac{\pi}{2 D} V,
$$

where $V=\int_{-D / 2}^{D / 2} A(s) d s$ is the volume of material (i.e., solid ice) in the particle. This ensures that the integral of the first term is equal to the volume of the particle [since the integral of the term in square brackets in Eq. (3) in the range $\pm D / 2$ is $2 D / \pi$ ]. The first term may then be subtracted from $A(s)$ and a Fourier transform performed on the residual to compute the terms $a_{j}^{\prime}$ and $a_{j}^{\prime \prime}$ to whatever order [represented by $n$ in Eq. (3)] is required. Note that terms with index $j$ correspond to variations with a wavelength of $D / j$.

This decomposition has been performed on each particle in the database of aggregates of bullet rosettes to obtain $a_{0}, a_{j}^{\prime}$, and $a_{j}^{\prime \prime}$ for each of the three directions: 


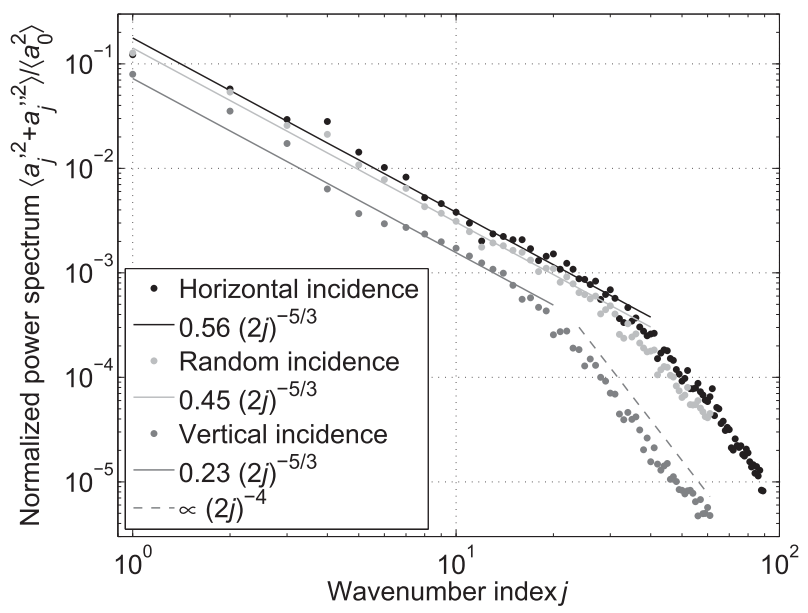

FIG. 3. The dots are normalized power spectra of the internal structure of the horizontally aligned aggregates of bullet rosettes discussed in section $2 \mathrm{~b}$, computed using the decomposition in Eq. (3) and averaged over the database of particles. The three spectra correspond to the horizontal direction, vertical direction, and averaged over all directions. The solid lines indicate fits to the spectra at scales larger than individual monomers (here corresponding to $j<20$ ) of the form in Eq. (5), using the coefficients in Table 1 . The dashed line indicates that the structure of the particles at scales smaller than individual monomers follows a power law with a slope of -4 .

$x, y$, and $z$. The dots in Fig. 3 depict normalized power spectra of the form $\left\langle a_{j}^{\prime 2}+a_{j}^{\prime \prime 2}\right\rangle /\left\langle a_{0}^{2}\right\rangle$ as a function of $j$ for horizontal incidence (an average of the variables derived from directions $x$ and $y$ ), vertical (derived from the $z$ direction), and random incidence (an average of the variables derived from all three directions).

At scales larger than individual monomer crystals ( $j$ less than around 20 in these examples, corresponding to scales larger than $D / 20$ ), the power spectra all follow a single power law. This behavior indicates that the shape of the particles, as described by $A(s)$, is statistically self-similar or fractal. This result supports the fractal paradigm of Sorensen (2001), but by performing a power spectrum on fluctuations from the mean shape rather than by parameterizing the autocorrelation function as a power law, we have sidestepped the problem of having to define a cutoff function.

The solid lines in Fig. 3 represent fits of the following form:

$$
\left\langle a_{j}^{\prime 2}+a_{j}^{\prime \prime 2}\right\rangle /\left\langle a_{0}^{2}\right\rangle=\beta(2 j)^{-\gamma},
$$

where $\beta$ and $\gamma$ are coefficients of the fit; $\beta$ quantifies the "energy" (i.e., amplitude squared) of the fluctuations relative to $\left\langle a_{0}^{2}\right\rangle$, and $\gamma$ quantifies the rate at which energy decreases when moving to smaller scales. The reason for the " 2 "' in the $(2 j)^{-\gamma}$ term is so that $\beta=1$ has the effect of making the amplitude of the first cosine in the first term on the right-hand side of Eq. (3) scale in exactly the same way as the terms in the summation. The best-fit values of $\beta$ for all three aggregate types are shown in Table 1, while $\gamma$ appears to have a value indistinguishable from the Kolmogorov value of $5 / 3$ for all three curves and all three aggregate types. The appearance of the Kolmogorov value in this context is surprising given that aggregation is a random process and in these simulations did not involve turbulence.

At scales smaller than individual monomer crystals ( $j$ larger than around 30 in these examples), the exponent appears to be closer to -4 ; this corresponds to the scale of solid ice within the monomer. Since all monomers are solid ice on very small scales, the same exponent should be found for all monomers, and indeed it is also found for aggregates of plates. A slight difference is that for aggregates of plates there is a more gradual transition between the slopes of $-5 / 3$ and -4 than for aggregates of bullet rosettes or columns.

\section{Backscatter of an ensemble of aggregates}

\section{a. Derivation of equation for backscatter cross section}

In this section we derive a formula for the ensembleaverage backscatter cross section $\left\langle\sigma_{b}\right\rangle$ of a population of aggregate particles of the same size (i.e., the same value of $D$ ) but with different shapes. Their shapes are assumed to be statistically self-similar in the sense described in the previous section. We first evaluate the integral in Eq. (1), which, for convenience, we define as

$$
I=\int_{-D / 2}^{D / 2} A(s) \exp (i 2 k s) d s
$$

for a single particle whose $A(s)$ function has been decomposed as in Eq. (3). This is achieved by replacing the sum of a cosine and a sine wave in Eq. (3) as follows:

$$
\begin{aligned}
& a_{j}^{\prime} \cos \left(\frac{2 \pi j s}{D}\right)+a_{j}^{\prime \prime} \sin \left(\frac{2 \pi j s}{D}\right) \\
& \quad=\frac{1}{2}\left[a_{j} \exp \left(-i \frac{2 \pi j s}{D}\right)+a_{j}^{*} \exp \left(i \frac{2 \pi j s}{D}\right)\right],
\end{aligned}
$$

where $a_{j}=a_{j}^{\prime}+i a_{j}^{\prime \prime}$ and $a_{j}^{*}$ is the complex conjugate of $a_{j}$. Note that although the right-hand side of Eq. (7) introduces imaginary terms, they cancel to leave the real function on the left-hand side, and the presence of exponentials is much easier to deal with when working out the integral in Eq. (6), which evaluates as 


$$
\begin{aligned}
I= & a_{0} \cos (k D)\left[\left(1+\frac{\kappa}{3}\right)\left(\frac{1}{2 k+\pi / D}-\frac{1}{2 k-\pi / D}\right)-\kappa\left(\frac{1}{2 k+3 \pi / D}-\frac{1}{2 k-3 \pi / D}\right)\right] \\
& -\sum_{j=1}^{n}(-1)^{j} \sin (k D)\left[i a_{j}^{\prime}\left(\frac{1}{2 k+2 \pi j / D}+\frac{1}{2 k-2 \pi j / D}\right)+a_{j}^{\prime \prime}\left(\frac{1}{2 k+2 \pi j / D}-\frac{1}{2 k-2 \pi j / D}\right)\right] .
\end{aligned}
$$

To derive a formula for $\left\langle\sigma_{b}\right\rangle$, it can be seen from Eq. (1) that the task is to find an expression for $\left\langle|I|^{2}\right\rangle$. Equation (8) has $2 n+1$ terms on the right-hand side, one corresponding to each " $a$ " coefficient, so taking the square leads to a large number of cross-multiplication terms.

To simplify the situation, we assume that the phase of the fluctuation at a particular scale is random. This has a number of consequences that can be expressed mathematically and is justified a posteriori by the good agreement between the resulting equation with direct application of Eq. (1) to the ensemble of simulated aggregates. First, it means that the ensemble average of the amplitudes of the individual sine and cosine waves is zero (since the amplitudes can be of either sign):

$$
\left\langle a_{j}^{\prime}\right\rangle=\left\langle a_{j}^{\prime \prime}\right\rangle=0
$$

Second, the randomness of the fluctuations means that there is no correlation between the amplitude of the sine and cosine terms in Eq. (3):

$$
\left\langle a_{j}^{\prime} a_{j}^{\prime \prime}\right\rangle=0 .
$$

Third, it means that the phase and amplitudes of the fluctuation at one scale are uncorrelated with those at another scale:

$$
\left\langle a_{j}^{\prime} a_{k}^{\prime}\right\rangle=\left\langle a_{j}^{\prime \prime} a_{k}^{\prime \prime}\right\rangle=0
$$

where $j \neq k$.

The result of Eqs. (9)-(11) is that when we square Eq. (8) and insert it into Eq. (1), the ensemble average of all the cross-multiplication terms is zero, so in practice we only need to square the $2 n+1$ terms individually. Thus, we obtain the mean backscatter cross section of an ensemble of aggregates:

$$
\begin{aligned}
\left\langle\sigma_{b}\right\rangle= & \frac{9 \pi k^{4}|K|^{2} V^{2}}{16}\left\{\cos ^{2}(x)\left[\left(1+\frac{\kappa}{3}\right)\left(\frac{1}{2 x+\pi}-\frac{1}{2 x-\pi}\right)-\kappa\left(\frac{1}{2 x+3 \pi}-\frac{1}{2 x-3 \pi}\right)\right]^{2}\right. \\
& \left.+\beta \sum_{j=1}^{n}(2 j)^{-\gamma} \sin ^{2}(x)\left[\frac{1}{(2 x+2 \pi j)^{2}}+\frac{1}{(2 x-2 \pi j)^{2}}\right]\right\}
\end{aligned}
$$

where $x=k D$. We refer to this model as the self-similar Rayleigh-Gans approximation (SSRGA). This expression produces the radar backscatter cross section with units of area. In the small-particle limit, the term in braces tends to $4 / \pi^{2}$ and the whole expression reduces to the Rayleigh approximation. In a numerical implementation of this formula we need to be careful with the denominators of some of the terms going to zero, but with $\cos$ or $\sin ^{2}$ in the numerator, the result is finite. Specifically the terms $\cos (x) /(2 x-\pi), \cos (x) /(2 x-3 \pi)$, and $\sin ^{2}(x) /(2 x-2 \pi j)^{2}$ become $-1 / 2,1 / 2$, and $1 / 4$, respectively, in the limit of their denominators tending to zero.

Note that because Eq. (12) only uses the coefficients of a single power law, it is only applicable when the wavelength is longer than the size of individual monomers. In the case of ice particles, Schmitt and Heymsfield (2014) reported ice monomer sizes up to $250 \mu \mathrm{m}$, much smaller than the wavelength of any meteorological radar. Thus, it is valid to neglect the steeper part of the power spectrum in Fig. 3 for current applications to snow. Should Eq. (12) ever be applied in a regime where the wavelength is smaller than individual monomers, then in principle the summation in Eq. (12) could be split into two ranges of $j$, one representing the aggregate structure and the other representing the monomer structure, with Fig. 3 suggesting that the latter should have an exponent of -4 .

\section{b. Evaluation of new equation for single particle sizes}

We now evaluate Eq. (12) for the mean backscatter cross section of an ensemble of aggregates of the same size, by comparing it with the mean of explicit application of the Rayleigh-Gans equation given by Eq. (1) to the ensemble of aggregates of bullet rosettes discussed in section 2b. The results are shown in Fig. 4 for both horizontally and vertically looking radars. Comparing the solid and dashed black lines, we see that the equation performs very well up to $k D_{x} \simeq 100$ and $k D_{z} \simeq 50$. At larger sizes or smaller wavelengths, corresponding to 

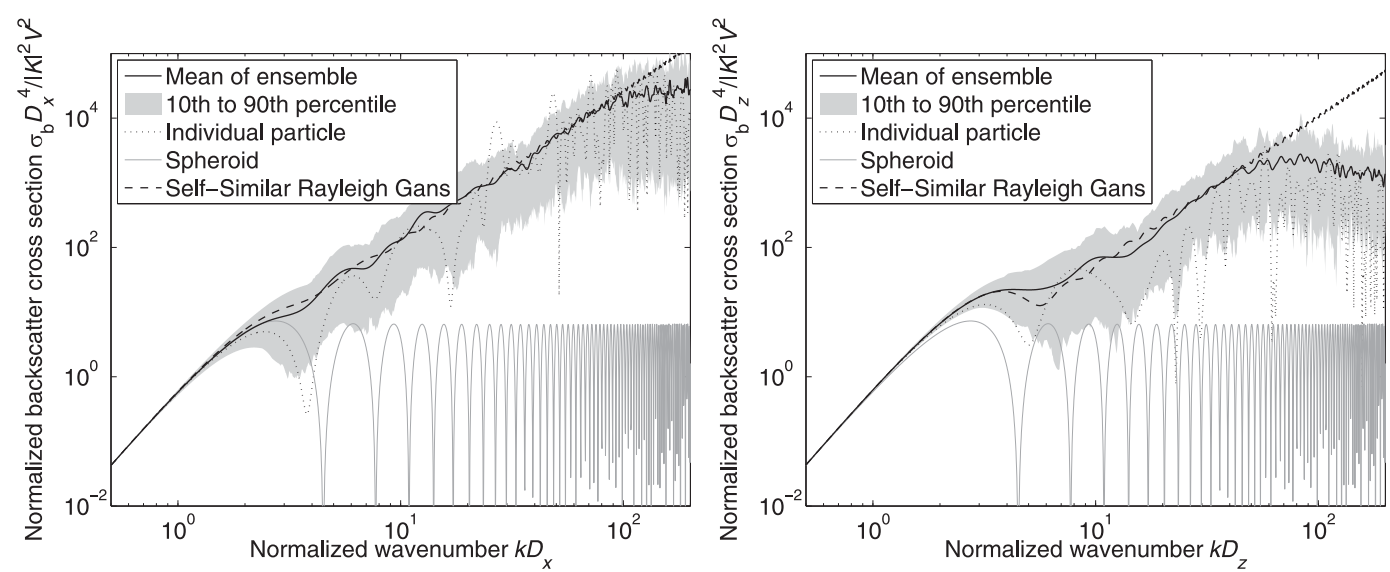

FIG. 4. Comparison of the mean backscatter cross section (solid line) and its spread (gray region) of the ensemble of horizontally oriented aggregates of bullet rosettes discussed in section $2 \mathrm{~b}$, computed by direct application of Eq. (1), to the equation derived in this paper given by Eq. (12) (dashed line). The dotted line shows the cross section of the individual particle depicted in Fig. 1, while the gray solid line shows the backscatter of a homogeneous ice-air spheroid with the same mass as the aggregate and same dimension in the direction of propagation of radiation. The results for (left) horizontally propagating radiation and (right) vertically propagating radiation (so using numbers from the first and second rows in Table 1, respectively) are shown. The normalization of the two axes makes the results applicable to all particle sizes, densities, and dielectric constants, provided that the conditions are met for applicability of the Rayleigh-Gans approximation.

the wavelength being smaller than the individual monomer crystals from which the aggregates are composed, the actual backscatter drops below that predicted by Eq. (12). This corresponds to the change in slope in Fig. 3. Note that the specific location of the change in slope depends on the relative size of the overall aggregates compared to the individual monomers. For relatively larger aggregates (those composed of many more monomers), the new equation would be applicable up to larger values of $k D$. However, the essential rule is that the shortest wavelength for which the new equation is applicable is approximately equal to the maximum monomer size, so for the Schmitt and Heymsfield (2014) value of $250 \mu \mathrm{m}$, the equation would be applicable up to frequencies of around $1200 \mathrm{GHz}$.

There are other small deviations between the two lines, the largest being at $k D_{z} \simeq 6$. This is believed to be because the assumptions made in section $3 \mathrm{a}$, which, while good, are not being perfectly satisfied (i.e., that the phase of the fluctuations at one scale either is not perfectly random or is not completely uncorrelated with the fluctuations at other scales).

Nonetheless, the equation is clearly a much better fit than the use of soft spheres or spheroids, which are shown by the solid gray lines. Consider 1-cm snowflakes observed by a $94-\mathrm{GHz}$ radar, which correspond to $k D \simeq$ 20: Fig. 4 shows that the soft spheroid assumption underestimates the backscatter cross section by around a factor of 40 for vertical incidence and 100 for horizontal incidence, similar to the difference found by Tyynelä et al. (2011) in calculations using the discrete dipole approximation. This difference is entirely due to the summation term in Eq. (12), which represents the fluctuations of mass within the particle on the scale of the wavelength that are ignored in the soft-spheroid assumption.

\section{The 94-GHz reflectivity factor for realistic snow distributions}

In this section we illustrate the impact of the new SSRGA scattering model for computations of radar reflectivity factor at the common frequency of $94 \mathrm{GHz}$, and hence the implications for retrievals in ice clouds and snowfall, by comparing it to soft sphere and softspheroid models for realistic particle size distributions. We first consider the model of Field et al. (2005), in which ice-particle size distributions are parameterized as a function of temperature and ice water content. This model was derived from a large database of aircraftmeasured size distributions in midlatitude ice clouds and snow. We assume the mass-size relationship of Brown and Francis (1995), which was found by Hogan et al. (2006) and Hogan et al. (2012) to lead to very good agreement between collocated Rayleigh-scattering radar measurements and aircraft observations in clouds containing unrimed ice particles. While the computed reflectivity factor $Z_{94}$ does depend on the mass-size 


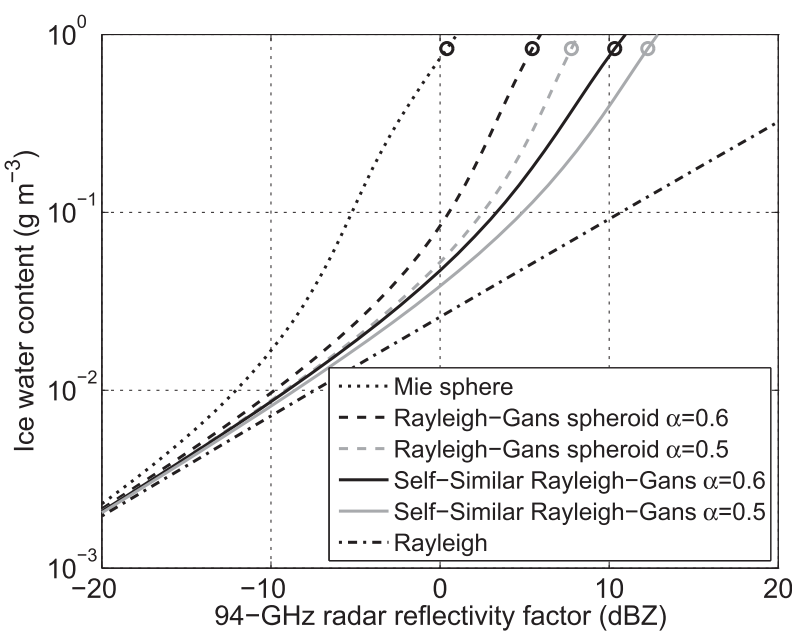

FIG. 5. The relationship between ice water content and 94-GHz radar reflectivity factor computed for the Field et al. (2005) size distributions at $0^{\circ} \mathrm{C}$ assuming the Brown and Francis (1995) masssize relationship. The sphere model assumed a homogeneous iceair mixture and used the Maxwell-Garnet mixing rule to compute the dielectric constant of the mixture for use in a Mie-scattering calculation. The oblate-spheroid model used the Rayleigh-Gans approximation given by Eq. (2) for aspect ratios of 0.6 and 0.5 . The self-similar Rayleigh-Gans model used Eq. (12) with the coefficients for aggregates of bullet rosettes or columns viewed at vertical incidence in Table 1, for the same two aspect ratios. The circles correspond to distributions with a median volumetric diameter of $7.3 \mathrm{~mm}$.

relationship, the difference in $Z_{94}$ between different scattering models does not depend on the prefactor of the mass-size relationship and is only weakly dependent on the exponent. Thus differences between models are essentially a function of particle size only, and these differences increase with particle size, so we consider a temperature of $0^{\circ} \mathrm{C}$, for which the Field et al. (2005) model predicts the largest particles.

Figure 5 depicts the relationship between ice water content and four scattering models: soft spheres, horizontally oriented soft spheroids, the new SSRGA equation given by Eq. (12) with the coefficients in Table 1 for aggregates of bullet rosettes or columns observed at vertical incidence, and Rayleigh scattering. Both the particle mass and horizontal extent $D_{\max }$ are identical between models. The assumed vertical extent of the particles is identical between the soft spheroid model and SSRGA. In this section we first consider the case of all particles having an aspect ratio of $\alpha=0.6$, which we regard as the "most likely" value based on previous observational and theoretical work (Korolev and Isaac 2003; Westbrook et al. 2004; Matrosov et al. 2005a; Hogan et al. 2012); these are shown by the black solid and dashed lines in Fig. 5. At the end of the section, we consider the impact of changing this value.
To get an idea of how far up the curves in Fig. 5 real snow distributions are found, we use the finding of Heymsfield et al. (2007) that if aircraft size distributions in snow are fitted by an exponential distribution of the form $N\left(D_{\max }\right)=N_{0} \exp \left(-\Lambda D_{\max }\right)$, the minimum slope parameter typically found is around $\Lambda=5 \mathrm{~cm}^{-1}$, corresponding to a maximum median volumetric diameter of $D_{0}=7.3 \mathrm{~mm}$. The Field et al. (2005) size distributions are not simple exponentials, but median volumetric diameter can be computed for any distribution and the circles in Fig. 5 show the points on the curves where $D_{0}=7.3 \mathrm{~mm}$. It can be seen that for particles this large, the SSRGA predicts $Z_{94}$ values that are $4.9 \mathrm{~dB}$ higher than the soft-spheroid approximation and $9.9 \mathrm{~dB}$ higher than the soft-sphere approximation. In terms of the effect on retrievals, we can see that if these curves were used in a lookup table, the retrieved ice water content (IWC) for an observed $Z_{94}$ of $5 \mathrm{~dB} Z$ would be a factor of 4.5 higher if soft spheroids were assumed compared to the more realistic SSRGA. For an observed $Z_{94}$ of $-5 \mathrm{dBZ}$, the overestimate would be around $25 \%$. While it is most straightforward to illustrate the impact on retrievals based on empirical relationships between IWC and $Z_{94}$ (possibly also including temperature), in principle any ice or snow retrieval making use of $Z_{94}$ measurements would be affected.

Aircraft observations of snow size distributions containing much larger particles have been reported, for example by Lawson et al. (1998) who measured size distributions that could be fitted by $N\left(D_{\max }\right)=$ $3 \times 10^{4} \exp \left(-100 D_{\max }\right)$ (SI units) with a maximum measured size of $5 \mathrm{~cm}$. Assuming again the Brown and Francis (1995) mass-size relationship, SSRGA predicts $Z_{94}=9.8 \mathrm{dBZ}$, which is $16 \mathrm{~dB}$ higher than that predicted by the soft-spheroid model and $24 \mathrm{~dB}$ higher than predicted assuming soft spheroids. Thus, it is clear that soft spheres and spheroids are totally inadequate for modeling the millimeter-wave backscatter from snowflakes.

The results so far in this section are for the most likely snowflake aspect ratio of 0.6 , but there is of course uncertainty in this value. The largest particles measured by Korolev and Isaac (2003) at temperatures warmer than $-15^{\circ} \mathrm{C}$ were reported to have a mean aspect ratio of 0.6 with a standard deviation of 0.1 (see their Fig. 11), and from a much smaller aircraft sample, Hogan et al. (2012) found $\alpha \simeq 0.5$ for $D_{\max } \simeq 4 \mathrm{~mm}$ (their Fig. 4). Therefore, we also compute the relationships assuming $\alpha=0.5$, shown by the gray lines in Fig. 5. The change in the IWC $-Z_{94}$ relationship due to this change in aspect ratio is also substantial, and for $Z_{94}<0 \mathrm{~dB} Z$, it is almost as large as the difference between the SSRGA and softspheroid models. Therefore, it is also important that 
future work can put a tighter bound on the aspect ratio of large snowflakes.

\section{Conclusions}

In this paper the self-similar Rayleigh-Gans approximation (SSRGA) has been introduced to derive an equation [Eq. (12)] for the backscatter cross section of ensembles of aggregate snowflakes at centimeter and millimeter wavelengths. It is applicable provided that the wavelength is longer than the individual monomer crystals within the aggregate, and that the mean density of the particle is low enough that the Rayleigh-Gans approximation (RGA) is applicable. This is valid for dry aggregates, but further work would be required to explore the extent to which riming might invalidate this assumption. The equation is much more convenient and far less computationally expensive than applying the discrete dipole approximation to a large number of simulated 3D particles. It is also much more accurate than the soft-spheroid approximation, predicting much higher backscatter cross sections for larger particles. This is important not only for snow remote sensing, but also potentially for radar retrievals in ice clouds.

A unique aspect to this work is that in characterizing the ice distribution function $A(s)$ in the viewing direction $s$, we first parameterize the mean structure and then describe the fluctuations from the mean in terms of a power law, finding a $-5 / 3$ exponent. The fact that a power law exists supports the fractal paradigm of Sorensen (2001), while avoiding the problems associated with using the autocorrelation function. It is intriguing that the exponent of the power law appears to take the Kolmogorov value and an interesting theoretical challenge for a future study would be to attempt to explain this based on the statistics of the aggregation process. It is also interesting that Leinonen et al. (2013) were able to provide a reasonable fit to the backscatter cross section when they did not use a power law. However, their autocorrelation model required the size of the monomers to be scaled up by a factor of as much as 7 , which thereby artificially added structure at scales intermediate between individual monomers and the aggregate as a whole. We argue that Fig. 3 is compelling evidence that a power law is the best way to describe the internal structure at the full range of scales from the monomers up to the entire aggregate and is likely to scale up better to aggregates of many more than 100 monomers.

We have provided the three coefficients that describe particle structure $(\beta, \gamma$, and $\kappa)$ for horizontal, vertical, and random incidence, and for aggregates composed of columns, plates, and bullet rosettes. Further work is required to estimate these parameters for aggregates of other crystal types such as dendrites, and to confirm that the coefficients are invariant for much larger aggregates than those considered here. While Westbrook et al. (2004) found universality in certain bulk properties of aggregates, there is a need to verify this for the specific case of these three coefficients. Furthermore, the results reported in this paper are potentially applicable to scattering by any type of aggregates (provided the RGA is applicable), such as optical scattering of aerosol aggregates (e.g., Sorensen 2001), but this might also require new coefficients to be derived.

Another avenue for future work arises because, for radiometry, the full scattering phase function is required rather than simply the backscatter cross section. In his book, van de Hulst (1957) showed how RGA could be applied to compute the scattering at any angle, so SSRGA could be extended to other scattering parameters. Note, however, that RGA is unable to predict polarimetric parameters since these depend on interactions between the dipoles within the particle, something that RGA explicitly neglects.

Acknowledgments. This work benefited from ESA Grant 4000104528/11/NL/CT and NERC Grant NE/ H003894/1. We thank Paul Field for useful discussions.

\section{REFERENCES}

Bohren, C. F., and D. R. Huffman, 1998: Absorption and Scattering of Light by Small Particles. Wiley, 544 pp.

Brown, P. R. A., and P. N. Francis, 1995: Improved measurements of the ice water content in cirrus using a total-water probe. J. Atmos. Oceanic Technol., 12, 410-414, doi:10.1175/ 1520-0426(1995)012<0410:IMOTIW>2.0.CO;2.

Draine, B. T., and P. J. Flatau, 1994: Discrete-dipole approximation for scattering calculations. J. Opt. Soc. Amer., 11A, 14911499, doi:10.1364/JOSAA.11.001491.

Field, P. R., R. J. Hogan, P. R. A. Brown, A. J. Illingworth, T. W. Choularton, and R. J. Cotton, 2005: Parameterization of ice particle size distributions for mid-latitude stratiform cloud. Quart. J. Roy. Meteor. Soc., 131, 1997-2017, doi:10.1256/ qj.04.134.

Heymsfield, A. J., G.-J. van Zadelhoff, D. P. Donovan, F. Fabry, R. J. Hogan, and A. J. Illingworth, 2007: Refinements to ice particle mass dimensional and terminal velocity relationships for ice clouds. Part II: Evaluation and parameterizations of ensemble ice particle sedimentation velocities. J. Atmos. Sci., 64, 1068-1088, doi:10.1175/JAS3900.1.

Hogan, R. J., P. R. Field, A. J. Illingworth, R. J. Cotton, and T. W. Choularton, 2002: Properties of embedded convection in warm-frontal mixed-phase cloud from aircraft and polarimetric radar. Quart. J. Roy. Meteor. Soc., 128, 451-476, doi:10.1256/ 003590002321042054.

, M. P. Mittermaier, and A. J. Illingworth, 2006: The retrieval of ice water content from radar reflectivity factor and temperature and its use in the evaluation of a mesoscale model. J. Appl. Meteor. Climatol., 45,301-317, doi:10.1175/JAM2340.1. 
, L. Tian, P. R. A. Brown, C. D. Westbrook, A. J. Heymsfield, and J. D. Eastment, 2012: Radar scattering from ice aggregates using the horizontally aligned oblate spheroid approximation. J. Appl. Meteor. Climatol., 51, 655-671, doi:10.1175/ JAMC-D-11-074.1.

Korolev, A., and G. Isaac, 2003: Roundness and aspect ratio of particles in ice clouds. J. Atmos. Sci., 60, 1795-1808, doi:10.1175/1520-0469(2003)060<1795:RAAROP > 2.0.CO;2.

Lawson, R. P., R. E. Stewart, and L. J. Angus, 1998: Observations and numerical simulations of the origin and development of very large snowflakes. J. Atmos. Sci., 55, 3209-3229, doi:10.1175/1520-0469(1998)055<3209:OANSOT>2.0.CO;2.

Leinonen, J., D. Moisseev, and T. Nousiainen, 2013: Linking snowflake microstructure to multi-frequency radar observations. J. Geophys. Res. Atmos., 118, 3259-3270, doi:10.1002/ jgrd.50163.

Levizzani, V., S. Laviola, and E. Cattani, 2011: Detection and measurement of snowfall from space. Remote Sens., 3, 145166, doi:10.3390/rs3010145.

Liu, G., 2008: Deriving snow cloud characteristics from CloudSat observations. J. Geophys. Res., 113, D00A09, doi:10.1029/ 2007JD009766.

Matrosov, S. Y., 1992: Radar reflectivity in snowfall. IEEE Trans. Geosci. Remote Sens., 30, 454-461, doi:10.1109/36.142923.

— , A. J. Heymsfield, and Z. Wang, 2005a: Dual-frequency radar ratio of nonspherical atmospheric hydrometeors. Geophys. Res. Lett., 32, L13816, doi:10.1029/2005GL023210.

—, R. F. Reinking, and I. V. Djalalova, 2005b: Inferring fall altitudes of pristine dendritic crystals from polarimetric radar data. J. Atmos. Sci., 62, 241-250, doi:10.1175/ JAS-3356.1.
Petty, G. W., and W. Huang, 2010: Microwave backscatter and extinction by soft ice spheres and complex snow aggregates. J. Atmos. Sci., 67, 769-787, doi:10.1175/2009JAS3146.1.

Pruppacher, H. R., and J. D. Klett, 1997: Microphysics of Clouds and Precipitation. Kluwer, $954 \mathrm{pp}$.

Sassen, K., 1977: Ice crystal habit discrimination with the optical backscatter depolarization technique. J. Appl. Meteor., 16, 425431, doi:10.1175/1520-0450(1977)016<0425:ICHDWT>2.0.CO;2.

Schmitt, C. G., and A. J. Heymsfield, 2014: Observational quantification of the separation of simple and complex atmospheric ice particles. Geophys. Res. Lett., 41, 1301-1307, doi:10.1002/ 2013 GL058781.

Sorensen, C. M., 2001: Light scattering by aggregates: A review. Aerosol Sci. Technol., 35, 648-687, doi:10.1080/02786820117868.

Tyynelä, J., J. Leinonen, D. Moisseev, and T. Nousiainen, 2011: Radar backscattering from snowflakes: Comparison of fractal, aggregate, and soft spheroid models. J. Atmos. Oceanic Technol., 28, 1365-1372, doi:10.1175/JTECH-D-11-00004.1.

C. D. Westbrook, D. Moisseev, and T. Nousiainen, 2013: Applicability of the Rayleigh-Gans approximation for scattering by snowflakes at microwave frequencies in vertical incidence. J. Geophys. Res. Atmos., 118, 1826-1839, doi:10.1002/jgrd.50167.

van de Hulst, H. D., 1957: Light Scattering by Small Particles. Wiley and Sons, $470 \mathrm{pp}$.

Westbrook, C. D., R. C. Ball, P. R. Field, and A. J. Heymsfield, 2004: Universality in snowflake aggregation. Geophys. Res. Lett., 31, L15104, doi:10.1029/2004GL020363.

,-- , and,- 2006 : Radar scattering by aggregate snowflakes. Quart. J. Roy. Meteor. Soc., 132, 897-914, doi:10.1256/ qj.05.82. 